\title{
RELATÓRIO ANUAL DE GESTÃO - 2007
}

1. Informação sobre distribuição (assinaturas, permutas, bibliotecas do Brasil e do Exterior etc.), incluindo, obrigatoriamente, o envio gratuito às bibliotecas de todos os programas de pós-graduação da área, em se tratando de periódico impresso

Circulação da Revista de Ciências da Administração - 2007

\begin{tabular}{|l|c|c|}
\hline \multicolumn{1}{|c|}{$\begin{array}{c}\text { Circulação da } \\
\text { RCA }\end{array}$} & Quantidade & Percentual \\
\hline Assinaturas & 27 & $12 \%$ \\
\hline Permutas & 52 & $23,11 \%$ \\
\hline $\begin{array}{l}\text { Bibliotecas do } \\
\text { Brasil }\end{array}$ & 83 & $32,44 \%$ \\
\hline $\begin{array}{l}\text { Bibliotecas do } \\
\text { Exterior }\end{array}$ & 9 & $4 \%$ \\
\hline $\begin{array}{l}\text { Bibliotecas dos } \\
\text { programas de Pós- } \\
\text { graduação em } \\
\text { administração }\end{array}$ & 64 & $28,44 \%$ \\
\hline Total & 235 & \\
\hline
\end{tabular}

2. Número de artigos submetidos, rejeitados, retirados, aceitos e em avaliação

\begin{tabular}{|l|l|l|}
\hline \multirow{2}{*}{\multicolumn{1}{c|}{$\begin{array}{c}\text { Número de } \\
\text { artigos }\end{array}$}} & \multicolumn{2}{c|}{ Janeiro a dezembro de 2007 } \\
\cline { 2 - 3 } Quantidade & \multicolumn{1}{c|}{ Percentual } \\
\hline Submetidos & 100 & $100 \%$ \\
\hline Rejeitados & 70 & $70 \%$ \\
\hline Retirados & 1 & $1 \%$ \\
\hline Aceitos & 10 & $10 \%$ \\
\hline Em avaliação & 19 & $19 \%$ \\
\hline Total & 100 & \\
\hline
\end{tabular}

3. Intervalo médio entre o recebimento, aprovação e publicação dos textos publicados nas revistas 17, 18 e 19 de 2007.

\begin{tabular}{|c|c|}
\hline Revistas de $\mathbf{2 0 0 7}$ & Tempo Médio \\
\hline n. 17 & 9,3 meses \\
\hline n. 18 & 11,7 meses \\
\hline n. 19 & 10,3 meses \\
\hline Tempo Médio Total & $\mathbf{1 0 , 4}$ meses \\
\hline
\end{tabular}

4. Nominata dos consultores ad hoc com filiação institucional completa

NOMINATA DOS AVALIADORES - 2007

Adelaide Maria Bogo Schmitt Adroaldo Lazzarotto

Affonso Henriques De Azevedo Nogueira
UNIVILLE- Universidade da Região de Joinville UNISINOS- Universidade do Vale do Rio dos Sinos

UNB - Universidade de Brasília 
Alberto Borges Matias

Alceu Souza

Alexandre Mendes Nicolini

Allan Augusto Platt

Alessandra de Linhares Jacobsen

Alexandre Marino Costa

Altair Borgert

Altamir da Silva Souza

Amélia Silveira

Ana Akemi Ikeda

Ana Alice Vilas Boas

Ana Augusta Ferreira de Freitas

Ana Maria Romano Carrao

Ana Maria Roux Valentini

Coelho Cesar

André Carlos Martins Menck

André Luiz Carvalhal da Silva

Andressa Sasaki Vasques

Pacheco

Angelise da Conceição

Valladares

Ângelo Antônio Cavalcante

Martins

Anielson Barbosa da Silva

Antônio Carlos Giuliani

Antônio Del Maestro Filho

Antônio Domingos Padula

Antônio Moreira de Carvalho

Neto

Carlos Loch

Carlos Ricardo Rossetto

Carlos Roberto Sanchez Milani

Carlos Yorghi Khoury

Catarina Cecília Odelius

César Augusto Tejera De Ré

Cláudia Rosa Acevedo

Cléber Carvalho de Castro

Cleverson Renan da Cunha

Dalila Alves Corrêa

Daniel Augusto de Souza

Daniel Augusto Moreira

Denise Del Pra Netto Machado

Elisa Yoshie Ichikawa

Emerson Wagner Mainardes

Enise Barth Teixeira

Esperidião Amin Helou Filho

Ester Eliane Jeunon

Eva Stal

Evelyn Maria Boia Baptista

Fernando Antônio Ribeiro Serra

Francisco Antônio Serralvo

Francisco Edson Bezerra de Brito

Júnior

Françoise Dominique Valéry

Gilberto de Oliveria Moritz
USP/RP- Universidade de São Paulo

PUC/PR- Pontifícia Universidade Católica do Paraná

UFBA - Universidade Federal da Bahia

UFSC- Universidade Federal de Santa Catarina

UFSC - Universidade Federal de Santa Catarina

UFSC - Universidade Federal de Santa Catarina

UFSC- Universidade Federal de Santa Catarina

FURG - Fundação Universidade Federal do Rio Grande

FURB - Fundação Universidade Regional de Blumenau

USP - Universidade de São Paulo

UFRRJ- Universidade Federal Rural do Rio de Janeiro

UECE - Universidade Estadual do Ceará

UNIMEP- Universidade Metodista de Piracicaba

UPM- Universidade Presbiteriana Mackenzie

UFU - Universidade Federal de Uberlândia

UFRJ- Universidade Federal do Rio de Janeiro

UFSC- Universidade Federal de Santa Catarina

Fundação Jorge Duprat Figueiredo de Segurança e Medicina do Trabalho

UFPE - Universidade Federal de Pernambuco

UnP - Universidade Potiguar

UNIMEP - Universidade Metodista de Piracicaba

UFMG- Universidade Federal de Minas Gerais

UFRGS - Universidade Federal do Rio Grande do Sul

PUC/MG - Pontifícia Universidade Católica de Minas Gerais

UFSC - Universidade Federal de Santa Catarina

UNIVALI - Universidade do Vale do Itajaí

UFBA - Universidade Federal da Bahia

UFBA - Universidade Federal da Bahia

UnB- Universidade Nacional de Brasília

UFRGS- Universidade Federal do Rio Grande do Sul

UNINOVE - Centro Universitário Nove de Julho

UFLA - Universidade Federal de Lavras

UFPR - Universidade Federal do Paraná

UNIMEP - Universidade Metodista de Piracicaba

UNIVALI - Universidade do Vale do Itajaí

USP - Universidade de São Paulo

FURB - Fundação Universidade Regional de Blumenau

UEM - Universidade Estadual de Maringá

FURB - Fundação Universidade Regional de Blumenau

UNIJUI- Universidade Regional do Noroeste do Estado do Rio

Grande do Sul

UFSC - Universidade Federal de Santa Catarina

UNIHORIZONTES- Faculdade Novos Horizontes

UNINOVE - Centro Universitário Nove de Julho

UFRGS- Universidade Federal do Rio Grande do Sul

UNISUL - Universidade do Sul de Santa Catarina

PUC/SP - Pontifícia Universidade Católica de São Paulo

UFPE- Universidade Federal de Pernambuco

UFRN- Universidade Federal do Rio Grande do Norte

UFSC - Universidade Federal de Santa Catarina 
Heloísa Márcia Pires

Henrique Luiz Correa

Herbert Kimura

Ivone Freire Costa

Jansen Maia Del Corso

João Adamor Dias Neves

João José Cândido da Silva

José Afonso Mazzon

José Antônio Nicolau

José Arimatés de Oliveira

José Carlos Barbieri

José Hamilton Maturano Cipolla

José Henrique de Faria

José Márcio de Castro

Leonardo Guerra de Rezende

Guedes

José Ricardo Costa de Mendonça

Leonel Cezar Rodrigues

Lia Hasenclever

Lilian Soares Outtes Wanderley

Luciana Marques Vieira

Luciano Munck

Luciano Zille Pereira

Luís Antônio Rabelo Cunha

Luís Moretto Neto

Luiz Carlos Duclós

Magnus Luiz Emmendoerfer

Maísa de Souza Ribeiro

Manuel Portugal Ferreira

Marcelo Álvaro da Silva Macedo

Marcelo Deschamps

Marcelo Gattermann Perin

Marcos Antônio de Souza

Marcos Baptista Lopez Dalmau

Marcus Vinícius Andrade de

Lima

Maria Ceci Araújo Misoczky

Maria de Lourdes Bacha

Maria Ester de Freitas

Maria Tereza Saraiva de Souza

Mário de Souza Almeida

Marlusa Gosling

Maurício Roque Serva de

Oliveira

Milton de Abreu Campanario

Nadia Kassouf Pizzinatto

Newton Carneiro Affonso da

Costa Júnior

Nilma Morcerf de Paula

Oscar Dalfovo

Osvaldo Elias Farah

Paulo de Paula Baptista

Paulo Fernando Burlamaqui

Paulo Sérgio Ceretta

Patrícia Vendramini
UFRJ - Universidade Federal do Rio de Janeiro

FVG - Fundação Getúlio Vargas

UPM - Universidade Presbiteriana Mackenzie

UFBA - Universidade Federal da Bahia

PUC/PR- Pontifícia Universidade Católica do Paraná

UECE- Universidade Estadual do Ceará

UFSC - Universidade Federal de Santa Catarina

USP - Universidade de São Paulo

UFSC - Universidade Federal de Santa Catarina

UFRN - Universidade Federal do Rio Grande do Norte

FVG - Fundação Getúlio Vargas

UNINOVE - Centro Universitário Nove de Julho

UFPR - Universidade Federal do Paraná

PUC/MG - Pontifícia Universidade Católica de Minas Gerais

UFGO - Universidade Federal de Goiás

UFPE - Universidade Federal de Pernambuco

UNINOVE - Centro Universitário Nove de Julho

UFRJ- Universidade Federal do Rio de Janeiro

UFPE - Universidade Federal de Pernambuco

UNISINOS - Universidade do Vale do Rio dos Sinos

UEL - Universidade Estadual de Londrina

UFMG- Universidade Federal de Minas Gerais

FAMETRO- Faculdade Metropolitana de Fortaleza

UFSC - Universidade Federal de Santa Catarina

PUC/PR- Pontifícia Universidade Católica do Paraná

UFMG - Universidade Federal de Minas Gerais

USP - Universidade de São Paulo

IPLEIRIA- Instituto Politécnico de Leiria- Escola Superior de

Tecnologia e Gestão

UFRRJ- Universidade Federal Rural do Rio de Janeiro

FURB - Universidade Regional de Blumenau

PUC/RS - Pontifícia Universidade Católica do Rio Grande do Sul

UNISINOS- Universidade do Vale do Rio dos Sinos

UFSC - Universidade Federal de Santa Catarina

UNISUL - Universidade do Sul de Santa Catarina

UFRGS - Universidade Federal do Rio Grande do Sul

UPM - Universidade Presbiteriana Mackenzie

FVG - Fundação Getúlio Vargas

UNINOVE - Centro Universitário Nove de Julho

UFSC - Universidade Federal de Santa Catarina

UFMG - Universidade Federal de Minas Gerais

UFSC - Universidade Federal de Santa Catarina

UNINOVE - Centro Universitário Nove de Julho

UNIMEP - Universidade Metodista Piracicaba

UFSC - Universidade Federal de Santa Catarina

ANHEMBI- Universidade Anhembi Morumbi

FURB - Fundação Universidade Regional de Blumenau

UNIMEP - Universidade Metodista Piracicaba

PUC/PR - Pontifícia Universidade Católica do Paraná

PUC/RS - Pontifícia Universidade Católica do Rio Grande do Sul

UFSM - Universidade Federal de Santa Maria

Faculdade Estacio de Sá de Santa Catarina 
Pedro Antônio de Melo

Pedro da Costa Araújo

Pedro Guilherme Kraus

Pedro José Steiner Neto

Ricardo de Souza Sette

Ricardo Silveira Martins

Roberto Giro Moori

Rodrigo Bandeira de Mello

Rolando Beulke

Rolando Juan Soliz Estrada

Rosalinda Chedian Pimentel

Rosilene Marcon

Rudimar Antunes da Rocha

Rui Otávio Bernardes de Andrade

Sandra Rolim Ensslin

Sérgio Alves

Suzane Strehlau

Tânia Casado

Valéria Maria Martins Judice

Valéria Silva da Fonseca

Valter Saurin

Victor Meyer Júnior

Vivaldo José Breternitz

Wilson Toshiro Nakamura
UFSC - Universidade Federal de Santa Catarina

UFSC - Universidade Federal de Santa Catarina

IBI- International Business Institute of Americas.

UFPR - Universidade Federal do Paraná

UFLA - Universidade Federal de Lavras

UFMG- Universidade Federal de Minas Gerais

UPM - Universidade Presbiteriana Mackenzie

UNIVALI - Universidade do Vale do Itajai

ULBRA - Universidade Luterana do Brasil

UFSM - Universidade Federal de Santa Maria

UNAERP- Universidade de Ribeirão Preto

UNIVALI - Universidade do Vale do Itajaí

UFSC - Universidade Federal de Santa Catarina

UFRRJ- Universidade Federal Rural do Rio de Janeiro

UFSC - Universidade Federal de Santa Catarina

UFPE - Universidade Federal de Pernambuco

ESPM- Escola Superior de Propaganda e Marketing

USP- Universidade de São Paulo

FDC- Fundação Dom Cabral.

PUC/PR - Pontifícia Universidade Católica do Paraná

UFSC - Universidade Federal de Santa Catarina

PUC/PR - Pontifícia Universidade Católica do Paraná

UPM - Universidade Presbiteriana Mackenzie

UPM - Universidade Presbiteriana Mackenzie 